\title{
Zur Thränensackexstirpation.
}

\author{
Von \\ Dr. P. Schreiber, \\ Assistenzarzt an der Graefe'schen Augenheilanstalt in Halle.
}

Seit Berlin im Jahre 1868 in der II. Sitzung der ophthalmolog. Gesellschaft*) der Exstirpation des Thränensackes das Wort geredet hat, ist wunderbarer Weise von einer Annahme seiner wohlzubeherzigenden Vorschläge nur wenig bekannt geworden. Die neuesten Lehrbücher z. B. von Klein**) berühren diese Operation mit keiner Silbe; und Schirmer im Sammelwerk von Gräfe-Sämisch***) zieht diese Operation mit den Worten: „ist meist schwer auszuführen und oft von heftiger Entzündung gefolgt" in die unverdiente Vergessenheit hinein, aus der sie Berlin mit warmer Empfehlung herausreissen wollte. Mandelstamm's Auslöffelung $\dagger$ ) der Schleimhant mit Erhaltung der Durchgängigkeit des ganzen Thränenkanals ist kaum hier anzuführen. Die einzige wohlgemeinte Empfehlung erfuhr B erlin von Seiten Sämis eh's, der, wie aus der Dissertation Oertmann's fif) hervorgeht, die Excision der Thränensack-

*) Klin. Monatsblätter, Jahrg. 1868, p. 355.

**) Lehrbuch der Augenheilkunde 1881, p. 769.

***) VII. Band, Abschnitt XII, p. 42.

t) Centralbl. f. pract. Augenh. 1879, p. 178.

t十) Oertmann: Ueber die Behandlung der Thränensackerkrankungen. Inaug.-Dissert. Bonn 1875. 
schleimbaut fur die mehr ader weniger seltenen. Falle von Hydrops sacei lacrymalis und Stenose des Nasolacrymalkanals für indicirt hält und demgemäss vier diese Indicationen illustrirende Falle beschreiben lässt. Allein sehr ermuthigend zur Nachahmung ronnten weder Sämisch's noch Berlin's Fälle sein und zwar aus folgenden Gründen: Einmal schien die Schwierigkeit der Ausfuhrung der Operation in keinem rechten Verhältniss zu den Recidiven zu stehen; denn sowohl Berlin wie Sämisch haben Fälle zu verzeichnen, in denen sie sich genöthigt sahen, zum zweiten Male zu operiren. Ferner kann eine Nachbehandlungsdauer von mindestens drei Wochen - in den meisten Fällen sogar mehr - auch nicht gerade sehr zu dieser Operation einladen. Der Hauptgrund ist aber der, dass die Indicationsgrenzen für diese Operation viel zu eng gesteckt waren, als dass sie überbaupt häufig hätte in Frage kommen können. Auf Grund ron 40 in den letzten zwei und ein Viertel Jahren von Herrn Prof. Graefe ausgeführten Exstirpationen bin ich in Stande, sowohl für die Operationstechnik einige Vorschläge zu machen, wie auch namentlich, das Gebiet der Indicationen erheblich zu erweitern.

Was zunächst die Indicationen zu diesem operativen Eingriff anlangt, so sind natürlich in erster tinie die von Sämisch aufgestellten: „Obliteration des ductus nasolacrymalis" und "Hydrops sacci" zn acceptiren. Ausser diesen Indicationen wären aber noch folgende aufzustellen:

1) Sehr hartnäckige Dacryocystoblennorrhoe mit Stenose des canalis nasolacrymalis, die zwar noch sondirt werden kann, jedoch schon so lange besteht, dass bereits eine leichte Vorbuckelung der Thränensackgegend und Verdickung der Wandung zu Stande gekommen ist.

2) Fistula sacei lacrymalis. Namentlich die Fälle, in denen durch häufig recidivirende Phlegmonen die bedeckende Hant mit der Sackwandung narbig verwachsen und indurirt ist. 
3) Ueberhaupt langwieriger Thränensackkatarrh bei volikommener Durchgängigkeit des Ductus; da demselben meist als Ursache polypöse Wucherung der Sackschleimhautzellen resp. kleine gestielte Tumoren (Schleimhautpolypen) vorzuliegen pflegen.

Diese Ausdehnung der Indicationen erklärt die grosse Zahl der hier ausgeführten Exstirpationen; denn von dem in den letzten $2 \frac{1}{4}$ Jahren behandelten Krankenmaterial von 10000 Patienten waren mit Thränenleiden überhaupt behaftet 285 Personen, also 2,85\%. Davon litten speciell an Dakryocystoblennorrhoe 173 (102 weibl., 71 männl. Geschlechts, also Verhältniss 3:2), von denen 40 die Operation indicirt erscheinen liessen, also fast $24 \%$.

Die Operation wird nun in folgender Weise am liegenden, chloroformirten Patienten vorgenommen. Der Schnitt ca. $2 \mathrm{~cm}$ lang, $4 \mathrm{~mm}$ vom inneren Augenwinkel, wird ziemlich vertical, mit dem oberen Ende etwas nach vorn geneigt, vorsichtig nur durch die äussere Haut geführt, um nicht gleich die Sackwandung anzuschneiden, was für die Ausschälung recht ausgedehnter Thränensäcke mit verdickter Wandung von grosser Wichtigkeit ist, da man dieselben eventuell prall gefült mit Eiter wie ein Atherom herauspräpariren kann. Indem man nun vorsichtig mit Hakenpincetten die Wundränder lüftet und mit einem Scalpell unterminirt, kann man die vordere Wand des Sackes ziemlich leicht blosslegen, ohne dass man einen Zweig der angularis oder eine Anastomose der frontalis anschneidet. Jetzt lassen sich mit dreizackigen stumpfen Wundhaken die Wundränder zurückziehen, und der Operateur fasst den Sack mit der Hakenpincette und umschneidet denselben sich immer möglichst am Thränenbein haltend oder präparirt ihn mit kleiner Cowperscher Scheere heraus, bei welchem Act bei derOperation sich allerdings nicht vermeiden lässt, dass man irgend eins von oben genannten Gefässen anschneidet und eine ziemlich starke Blutung 
hervorruft. Etwaige zurückbleibende Reste werden dann schonungslos mit dem Volkmann'schen scharfen Löffel bis hart anf den Knochen herausgeschabt. Ebenso wird mit einem kleineren Caliber des eben genannten Löffels in den Thränennasenkanal eingegangen, so weit derselbe noch durehgängig ist, und die Schleimhaut in demselben gründlichst zerstört. Darauf Irrigation der Wunde mit $2 \%$ Carbolsäure und 3-6 Näthe, so dass eine vollkommene Vereinigung der Wundränder zu Stande kommt. Eine so ausgeführte ideal verlaufende Exstirpation würde mit Chloroformnarkose etwa 20-30 Minuten in Anspruch nehmen und zur Nachbehandlung höchstens 14 Tage erfordern. So ideal verlaufen zwar nicht alle Operationen. So stellt sich zum Beispiel bei der Exstirpation bedingt durch fistula sacci lacrymalis die Schwierigkeit entgegen, dass die bedeckende Haut durch die voraufgegangene Phlegmone sehr brüchig geworden ist, und daher beim Fassen mit der Pincette leicht ausreisst. Oft ist die Haut in diesen Fällen auch mit der Sackwandung innig verwachsen und daher schon schwerer zu präpariren. Schliesslich ist auch die Blutung bei diesen chronisch entzündlichen Geweben eine stärkere und verdeckt das Operationsterrain. In diesen Fällen mache man trotz Blutung ausgedehnte Anwendung des scharfen Löffels, der hier um so mehr wirkt, je brüchiger die Gewebe sind. Hat man dann den Knochen rein vor sich, so gemügt nach gründlicher Desinfection eine genaue Nath der vorher mit der Scheere adaptirten, zerklüfteten Wundrändèr, um die Blutung zu stillen, und eine Heilung per primam zu erzielen. Diese Fälle von fistula sacci lacrym. nach vorausgegangener oder noch bestehender Dacryocystitis phlegmonosa sind demnach für die Operation selbst die unangenehmsten, in ihren Erfolgen aber entschieden ebenso schön, wie die bei nicht entzündlichen Leiden operirten.

Die Nachbehandlung gestaltet sich sehr einfach. Sie 
besteht in mehrmaligem Wechseln des antiseptischen Verbandes (Borlint, mit 4\% Borsäure getränkt, darüber Wachstaffet, Carbolwatte und Binde) und Entfernen der Näthe am 2. resp. 3. Tage. Diese wohlvereinigte Wunde heilt stets per primam. Wundkrankheiten haben wir nie nach der Exstirpation zu beobachten Gelegenheit gehabt.

Zur Mlustration meiner Behauptungen lasse ich hier einige der prägnantesten Krankengeschichten folgen:

\section{Fall I (Journal-Nr. 76818).}

Marie L. ans Neuhaldensleben, 25 Jahre alt, ist seit 5 Jahren thränenleidend. Am linken Auge ist eine Narbe sichtbar, die sich vom Canthus internus ca. $1 \mathrm{~cm}$ weit nach unten hinzieht, leicht wulstig ist, und ein Ectropium des unteren Lides zur Folge gehabt hat. Im oberen Theile der Narbe nimmt man eine capillare Thränenfistel wahr. Die Hauptbeschwerden klagt jedoch Pat. über das rechto Auge, an dessen inneren Winkel sich seit 2 Jahren eine langsam wachsende Geschwulst gebildet hat, aus der Pat. zu Anfang noch nach der Nase und dem Conjunctivalsack hin Fiter hat ausdrücken können. Seit einem Jahre ist der Weg nach der Nase jedoch verlegt, und in der letzten Zeit hat Pat. auch nach dem Conjunctivalsack nur mit Mühe den Eiter entlecren können. Der Tumor, reichlich haselnussgross, fühlt sich prall elastisch an. Die Haut über demselben ist leicht verschieblich und nicht geröthet. Auf starken Druck entleert sich eine geringe Quantität Eiter nach dem Conjunctivalsack hin.

Am 25. Mai 1881 wird die Exstirpation des Sackes vorgenommen, der sich bequem, ohne ihn anzuschneiden wie ein Atherom herausschälen lässt, und wobei sich herausstellt, dass der ductus naolacrymalis total obturirt ist. Die Blutung ist unerheblich. Die Maasse des exstirpirten Sackes sind: Länge $23 \mathrm{~mm}$, Breite $15 \mathrm{~mm}$, Dicke der Sackwandung schwankt zwischen 4 und $5 \mathrm{~mm}$. Dureh 6 Näthe wird der etwa $213 \mathrm{~cm}$ lange Hautschnitt geschlossen, nachdem die Wunde vorher durch Carbolirrigation gereinigt war. Darauf antiseptischer Verband.

Am 28. Mai werden die Näthe entfernt. Der obere und untere Theil des Schnittes sind per primam ohne alle Reaction vernarbt. In der Mitte klaffen die Wundränder leicht und 
lassen auf Druck aus der Tiefe der durch die Operation gesetzten Höhle, die sich bereits vollständig mit Granulationen gefullt hat, etwas seröseitriges Secret ausdrücken. Dieser Zustand orhält sich noch bis zum 31. Mai, wo sich ein Schorf über dieser Stelle bildet, der sich bis zum 8. Juni vollständig losgestossen hat. Die Narbe ist jetzt fast unsichtbar und auf Druck unempfindlich. Pat. klagt absolut nicht über Thränen und wird nach 14 tägiger Bebandlung vorläufig entlassen.

\section{Fall II (Journal-Nr. 74799).}

Frau Elisabeth E. aus Barchau bei Burg, 39 Jahre ait, datirt ibr rechtsseitiges Thränenleiden von einer Erkältung, die sie sich vor 4 Jahren zugezogen hat. Ein Jahr später fühlte sie bereits eine Vorbuckelung in der Thränensackgegend. Auf Druck entleerte sich damals noch "Eiter in das Auge". Zuletzt liess sich das Secret jedoch nur noch nach der Nase hin ausdrücken und genirte die Pat. ungemein durch den unerträglichen Foetor. Stat, präs.: Starke Vorbuckelung - etwa kleine Haselnuss grosse Geschwnlst - in der Thränensackgegend. Resistenter, elastischer Tumor, über dem die nicht geröthete Haut leicht versehieblich ist, und aus dem sich nur sehr schwer nach der Nase zu. Eiter ausdrücken lässt. Sondenbehandlung wird nicht mehr versucht, sondern zur Exstirpation geschritten, sobald sich der Sack wieder prall gefüllt hat.

Am 24. November 1880 wird die Exstirpation des Sackes in toto mit dem ganzen eitrigen Inhalt ausgeführt, was sehr leicht gelingt, da die Blutung verhältnissmässig sehr gering ist. Die Wunde wird mit 3 Nathen geschlossen und antiseptisch behandelt. Die Maasse des extirpirten Sackes waren: Länge $2 \mathrm{~cm}$, Breite $1,1 \mathrm{~cm}$.

Am 27. November Entfernung der Nathe. Wunde per primam ohne alle Reaction geheilt.

Am 30. November wird Pat. - also nach 7 tägiger Behandlung - mit etwas Thränenträufeln und geringer Drackempfindlichkeit entlassen.

Diese beiden Beispiele mögen hinreichen, um den Werth der Exstirpation bei Hydrops sacci lacrym. und Stenose des ductus naso lacrym. klar zu stellen, und zugleich beweisen, wie wichtig es ist, die Sackwandungen. 
nicht anzuschneiden, sondern den Sack in toto herauszupräpariren. Erstens ist die Operation auf diese Weise viel leichter, als wenn man die Wandungen in einzelnen Fetzchen herauspräparirt. Zweitens ist auch der Erfolg ein viel sicherer, da derselbe zum grossen Theil davon abhängt, dass man nichts von der Sackwandung stehen lässt. Leider lässt sich das ideale Herauspräpariren nur in Fällen von hochgradigem Hydrops sacci lacrym. mit starker Wandverdickung ausführen, während man bei Exstirpation des Sackes wegen Fistula sacci und überhaupt bei langwieriger Dacryocystitis mit erhaltener Durchgängigkeit des Ductus die Operationsmethode etwas modificiren muss, wie die folgenden Beispiele zeigen.

\section{Fall III (Journal-No. 74906).}

Frau L. aus Wessmar bei Gröbers, 30 Jahre alt, klagt seit 莦 Jahr über Thränen. Seit vier Wochen hat sie die „Rose" in der Lidecke, die vor 14 Tagen zum Aufbruch gekommen ist. Bevor diese Phlegmone enstanden ist, soll die Thränensackgegend nicht geschwollen gewesen sein. Es handelt sich um eine Dacryocystitis phlegmonosa mit Fistelbildung.

Am 5. December 1880 Exstirpation des Sackes mit folgender Modification. In der gewöhnlichen Weise wird der Schnitt $2 \mathrm{~cm}$ lang jedoch mit Eroffnung des Sackes geführt. Darauf jedoch der ganze Sack mit dem scharfen Löffel ausgeräumt bis auf das Thränenbein. Ebenso wird die Schleimhaut aus dem Ductus naso lacrymalis mit kleinerem Löffel herausgekratzt; darauf werden die morschen Wundränder mit der Scheere abgetragen und durch drei Suturen vereinigt. Antiseptischer Verband. Die Heilung erfolgt per primam. Die Narbe ist nicht sehr wulstig, jedoch ist bei der Entlassung am 14. December der innere Augenwinkel etwas ödematös, und wird von der Pat. über geringes Thränenträufeln geklagt. Auf Druck lässt sich kein Secret nach dem Conjunctivalsack hin entleeren.

Pat. stellt sich am 25. Februar 1881 wieder vor. Die Narbe ist fast linear. Thränenschlanch und Sack vollständig

₹. Graefe's Archiv fitr Ophthalmologie. XXVII. 2. 
verödet. Es besteht keine Conj., trotzdem aber noch geringes Thränenträufeln.

\section{Fall IV (Journ.-No. 75801).}

Der Frau Louise B., 53 Jahre alt, aus Bennekenstein hat bereits über ein Jahr lang das linke Auge "voll Wasser gestanden". Martini 1880 ist es dann zu einer Phlegmone mit Fistelbildung gekommen. Patientin leidet an Stockschnupfen, und ist das Thränenleiden wohl darauf zurückzuführen.

Stat. präs.: Bohnengrosser Tumor in der linken Thränen. sackgegend mit gerötheten, schlecht versehieblichen Hautdecken, aus dem sich mit Mühe Secret nach dem Conjunctivalsack entleeren lässt.

Am 7. Marz 1881 Exstirpation. Der Schnitt, obwohl sehr vorsichtig geführt, eroffnet sofort den an einer Stelle sinnöss ausgebuchteten Thränensack. Relativ leicht ist die im Allgemeinen sehr verdickte vordere Wandung zu exstirpiren. Beim Herauspräpariren der hinteren Wandung wird die dureh den entzündlichen Process sehr erweiterte Art. angularis angeschnitten, wodurch eine so störende Blutung entsteht, dass eine Schieberpincette in Anwendung kommt. Die hintere Wandung wird darauf mit scharfem Löffel so gründlich entfernt, dass das Thränenbein an einer Stelle perforirt wird. Ausserdem wird die Schleimhaut des Thränennasencanals mit dem scharfen Löffel bearbeitet.

Die Blutung wird durch Anlegen von sechs Suturen gestillt. Heilung erfolgt per prim., so dass am 9. März die Suturen entfernt werden. Am 16. März wird Patientin mit geringem Thränenträufeln mit absolut strichförmiger, auf Druck nur wonig empfindlicher Narbe entlassen. Nach dem Conjunctivalsack hin lässt sich nichts von Secret entleeren.

\section{Fall V (Journ.-No. 76398).}

Anna H. aus Dörstewitz bei Lauchstaedt, 13 Jahre alt, klagt seit Herbst 1880 über Thränen des rechten Auges und Ansammlung von "Fiter in der Lidecke". Im Februar 1881 bildete sich „ein Geschwür" in der Thränensackgegend, das aufbrach, und seitdem besteht eine Thränenfistel. Die Thränensackgegend ist mässig prominent und zeigt Narben einer früheren Phlegmone. 
Am 7. April 1880 Exstirpation, bei der die Blutung ungewöhnlich stark ist. Das Herauspräpariren des Sackes ist sehr erschwert, da die verdickte Wandung sehr innig mit den Hautdecken verwachsen ist. Es wird daher sehr ausgedehnte Anwendung vom scharfen lü̈ffel gemacht. Die Wunde wird mit vier Suturen geschlossen und antiseptisch bebandelt. Von den Suturen reisst die in den inneren Lidwinkel gelegte am zweiten Tage aus, da das Gewebe sehr morsch war. Nichtsdestoweniger verheilt die Wunde innerhalb 14 Tagen so vollständig, dass Patient mit geringer Conj. und leichtem Tbränenträufeln entlassen werden kann. Nach dem Conjunctivalsack lässt sich kein Secret ausdrücken.

\section{Fall VI (Journ.-No. 75733).}

Fran Sophie G. aus Wulferstedt, 49 Jahr alt, kommt am 1. März 1881 in Behandlung mit lange bestehendem, prall elastischem Tumor, der sich nicht nach dem Conj.-Sack, dagegen nach der Nase hin entleeren lässt. Die Haut darüber ist nicht geröthet aber auch wenig verschieblich. Bei der Exstirpation, die an demselben Tage vorgenommen wird, zeigt es sich, dass die Tumorwandung doch sehr innig mit den Hautdecken verwachsen ist, und eine reine Herausschälung nicht möglich ist. Der Sack wird angesehnitten, wobei sich eine ziemliche Quantität Eiter ergiesst. Die abtragbaren Theile werden mit der Scheere abgekappt. Der Grund mit dem scharfen Löffel ausgekratzt. Darauf genane Desinfection und Suturen, die am 3. März entfernt werden, am 6. März wird Patientin mit etwas Conjunctivaleatarrh und leichter Epiphora geheilt entlassen.

Sehr einleuchtend spricht der folgende Fall für die Indication der Exstirpation bei hartnäckiger Dakyoeystoblennorrhoe trotz leichter Durchgängigkeit des ductus nasolacrym., die in diesem Falle durch einen kleinen glattwandigen, gestielten Tumor unterhalten war.

\section{Fall VII (Journ.-No. 75770).}

Frau B., 39 Jahr alt, aus Damsendorf b. Flechtingen sucht am 3. März 1881 mit linkseitigem frischen uleus serpens 
und rechtsseitigen alten Residuen desselben Leidens - nämlich grossem leucoma adhärens - unsere Hülfe auf. Beiderseits besteht Dacryocystoblennorrhoe mit gexinger Verdickung der Thränensackwandungen. Nach mehrwöchentlicher Behandlung wird das nlens serpens geheilt mit Hinterlassung eines grossen centralen Leucoms. Auch versehwindet die eitrige Secretion aus dem Thränensack der rechten Seite vollkommen auf die eingeleitete Sondenbehandlung; wäbrend links die Secretion weder auf Sondenbehandlung noch Einspritzungen ron Antiseptischen und Arg. nitr. Lösungen vermittelst Anel'scher Spritze sistirt. Es wird daher nach 7 wöchentlicher vergeblicher Behandlung am 26. April 1881 zur Exstirpation des linken Thränensackes gesehritten. Es wird zunächst versucht, denselben heraus zu präpariren, was jedoch wegen der ziemlich starken Blutung nur mit der vorderen Wandung gelingt. Beim Einschneiden in den Sack findet sich in demselben ein kleiner kugelrunder Tumor von der Grösse eines Hanfliornes. Derselbe ist vollkommen abgekapselt und hängt an einem sehr dümnen Stiele, der in dem Moment abreisst, als der Tumor mit der Pincette gefasst wird. Es liegt sehr nahe, diesen kleinen Tumor, der mikroskopisch untersucht aus stark vascularisirtem, rundzelligem Gewebe bestand, zu beschuldigen, die Eiterung im Thränensack so lange unterhaiten zu haben.

Nach 8 Tagen war Pat. so weit hergestellt, dass auch auf diesem Auge eine die optischen Verhaltnisse sehr verbessernde Iridectomie rorgenommen werden konnte.

Dieselbe Ursache für die Hartnäckigkeit der Thränensackeiterung liegt im folgenden Falle vor:

\section{Fall VIII (Journal-Nr. 73528).}

Der Knabe Reinhold B., 8 Jahre alt, aus Naundorf bei Alsleben, war bereits lange Zeit wegen linksseitiger Dakryocystoblennorrhoe, die häufig zu. Secretsretention führte, in Behandinng. Der Thrönensack war pathol, erweitert, der ductus lacrym. dabei gut durchgängig, aber schwer zu sondiren, da die Sonde, wie angenommen wurde, immer in einer Schleimhautfalte hăngen blieb. Dagegen zeigte sich bei dor am 6. August 1880 vorgenommenen Exstirpation, dass der Grund für dieses Hängenbleiben in einem Tumor zu suchen war, der beim Einschneiden in den Sack höehst unberraschend heraus- 
sprang. Der Tumor war etwa erbsengross, ovoid, glattwandig, leicht eindrückbar, blasenartig und schien vollständig abgekapselt zu sein. Bei genauerer Untersuchung ergab sich, dass derselbe ein kleines Gefässstämmchen zum Stiel gehabt hatte, und die mikroskopische Untersuchung stellte dieselbe morphologische Beschaffenheit - reich vaseularisirte Granulationsgeschwulst - wie im vorigen Falle fest.

Zum Schluss möchte ich mit einem Beispiel noch einmal auf die Wichtigkeit hinweisen, den ganzen Thränensack heraus zu präpariren und sich nicht etwa nur mit der vorderen Wand za begnügen:

\section{Fall IX (Journal-Nr. 74563).}

Frau Emilie 0, aus Merseburg, 36 Jahre alt, leidet seit einem Jahre an Dacryocystoblennorrhoe mit Retention des Secrets. Die rechte Thränensackgegend ist bereits bohnengross vorgebuckelt. Der Tumor fühit sich fest an, und nur auf starken Druck ist man im Stande das Secret nach dem Conjunctivalsack hin zu entleeren, das, wie die mikroskopische Untersuchung ergiebt, Eiterkörperchen, viel Mikrococcenhaufen und einige Epithelzellen enthält. In früherer Zeit ist das Secret nach Angabe der Pat. auch nach der Nase hin ausdrückbar gewesen. Die Haut ist leicht über dem Tumor verschieblich, und war nie phlegmonös geröthet.

Am 29. December 1880 Exstirpation, die von Anfang an äusserst blutig verläuft. In Folge dessen muss die ganze Operation, so zu sagen, im Dunkeln ansgeführt werden; denn trotz allen Tupfens mit Schwämmen war kein klares Operationsfeld - selbst auf Augenblicke nicht - zu erzielen. Es wurde unter diesen ungünstigen Verbältnissen zwar sehr nachdrücklich mit dem scharfen Löffel gearbeitet, nichtsdestoweniger blieb von der hinteren Wand ein kleines Stück stehen, was sich später sehr bemerkbar machte. Denn während sonst in spätestens 8 Tagen die Wundränder mit einander zu verwachsen pflegen, klaffte in diesem Falle die Wunde in der Mitte in ca. $\frac{1}{2} \mathrm{~cm}$ Ausdehnung, und es entleerte sich auf Druck eine ziemliche Quantität Eiter. Nach 3 Wochen war zwar die Hautwunde allerdings etwas walstig vernarbt, dagegen liess sich nach dem Conjunctivalsack hin etwas eitriges Secret ausdrücken. Auf ihren Wunseh wird Pat. vorläufig entlassen. Am 5. Fe- 
bruar 1881 stellt sich Pat, wieder vor mit einer Narbe, die einerseits nicht gerade allen cosmetischen Anforderungen genügt und ausserdem eine kleine fistulöse Oeffnnng enthält, die in einen ungefähr $1 \mathrm{~cm}$ langen Gang hineinführt. Aus dieser Oeffnung entleerte sich klare Thränenflüssigkeit.

Es handelt sich hier um einen Fall, der vollständig analog ist den von Berlin mehrfach beobachteten, und die Berlin dadurch zu deuten suchte, dass er dem Thränenzufluss die heilungstörende Rolle in diesen Fällen zuschrieb.*) Ich kann mich zu dieser Ansicht nicht bekehren, da ich sonst nie etwas von heilungsstörendem Einfluss der Thränenflüssigkeit in der Nachbehandlung unserer Exstirpationsfälle gesehen habe, glaube vielmehr dem Umstande die Schuld beimessen zu müssen, dass ein Stück blennorrhoisches Secret absondernder Schleimhaut stehen geblieben ist, welches natürlich keine Verwachsung mit gegenüberliegenden Wundfächen eingehen kann, sondern im Gegentheil im Stande ist, einen neuen Hohlraum herzustellen. Es kommt daher vor allen Dingen bei der Exstirpation des Thränensackes darauf an, die ganze Sackwandung heraus zu präpariren, bis man den blosliegenden Knochen des Thränenbeines sieht. Dies kann man auch erreichen trotz starker Blutung, die in den meisten Fällen nicht zu vermeiden ist, durch rigorose Anwendung des scharfen Löffels, mit dem man auch arbeiten kann, ohne dass man das Operationsfeld klar ubersieht. Auf diese Weise ist die Exstirpation des Thränensackes eine nicht zu schwer auszuführende Operation, die antiseptisch ausgeführt und nachbehandelt absolut ungefährlich, in kürzester Zeit zum Ziele führend und für die Patienten von keinen unangenehmen. Folgen begleitet ist.

*) 1. c., p. 360 . 